\title{
Model of Automobile Parts Sale Prediction Based on Nonlinear Periodic Gray GM(1,1) and Empirical Research
}

\author{
Lixiong Gong $\mathbb{D}^{1,2,3}$ and Canlin Wang ${ }^{4}$ \\ ${ }^{1}$ School of Mechanical Engineering, Hubei University of Technology, Hubei, Wuhan 430068, China \\ ${ }^{2}$ College of Mechanical Engineering, Chongqing University of Technology, Chongqing 400054, China \\ ${ }^{3}$ Hubei Key Lab of Manufacture Quality Engineering, Hubei, Wuhan 430068, China \\ ${ }^{4}$ Shenzhen Zhiboyi Enterprise Management Consulting Co., Ltd, Guangdong, Shenzhen 518172, China \\ Correspondence should be addressed to Lixiong Gong; herogong2001@sohu.com
}

Received 27 June 2019; Accepted 1 August 2019; Published 27 August 2019

Academic Editor: Anna M. Gil-Lafuente

Copyright (C) 2019 Lixiong Gong and Canlin Wang. This is an open access article distributed under the Creative Commons Attribution License, which permits unrestricted use, distribution, and reproduction in any medium, provided the original work is properly cited.

\begin{abstract}
The traditional predictive method cannot fully reflect the complex nonlinear characteristics and regularities of automobile and parts sales data, so the prediction precision is not high. The purpose of this paper is to propose the gray GM $(1,1)$ nonlinear periodic predictive model by introducing the seasonal variation index to improve predictive accuracy of the single GM(1,1) model. Firstly, the paper analyzes concept of $\operatorname{GM}(1,1)$ and then proposes the gray $\operatorname{GM}(1,1)$ nonlinear periodic predictive model to forecast automobile parts sales. The model algorithm used gray theory and accumulated technology to generate new data and set up unified differential equations to find the fitting curve of automobile parts sales prediction by the seasonal variation index to remove random elements. Lastly, the gray $\mathrm{GM}(1,1)$ nonlinear periodic predictive model is used for empirical analysis; the result of example shows that the model proposed in the paper is feasible. The superiority of the proposed predictive model compared with the single gray GM $(1,1)$ model is demonstrated. The reliability of this model is experienced by the accuracy test, which provides a theoretical guidance for the prediction of automobile part sales. And the average relative error is reduced by $8.52 \%$ compared with the single $\mathrm{GM}(1,1)$ model.
\end{abstract}

\section{Introduction}

In recent years, China's automobile industry has grown rapidly with the development of the automotive market. Profiting from the improvement of the level of information, sales data of the dealer are increasingly abundant. Therefore, it is very important to predict the sales of automobile parts, regardless of the policy makers of the automobile industry or the research of the market strategy of the automobile or parts manufacturers $[1,2]$. The traditional automobile parts sale predictive model is only adopted in single and simple mode, such as a time sequence prediction method that only considers the historical data. And the Delphi method with obvious subjectivity did not consider the change of future market trends and economic environment [3-5]. Other predictive methods simply considered time growth, like the linear regression model, random time series model, and exponential function model [6-9], which have only considered the data on a variety of trends and cannot fully reflect the complex nonlinear characteristics and regularities of automobile and parts sales data, so the prediction precision is not high. Therefore, it is necessary to accurately predict sales of automobile and its parts and build the appropriate sales predictive model to integrate complex, random, nonlinear, and time-varying information and data. At present, the gray theory has shown an extremely strong function in the recognition of information, transformation, processing, transmission, storage, control, and other aspects [10-12]. Zhicheng et al. [13] has analyzed the influences of various factors on heat transfer characteristics using the gray correlation theory. Memon et al. [14] presented to apply the combination of gray system theory and uncertainty theory to select the suppliers, in which the proposed supplier selection method not only selects the most appropriate suppliers but 


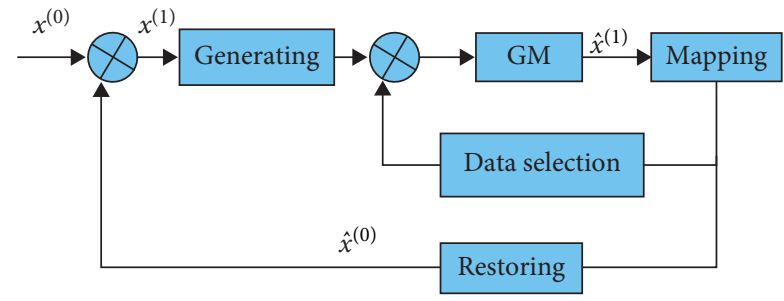

Figure 1: Data processing program.

also allocates optimal purchase quantity under stochastic and recognitive uncertainties. Zeng et al. [15] presented a multivariate gray model suitable for the sequence of ternary interval numbers and forecasted for China's electricity generation and consumer price index. A seasonal $\operatorname{GM}(1,1)$ model and gray modelling method $\operatorname{DGGM}(1,1)$ were proposed. Wang et al. $[16,17]$ predicted the seasonal fluctuations of the electricity consumption and the quarterly hydropower production in China. Li et al. [18] proposed the $\mathrm{GM}(1,1)$ predictive model, which realized the prediction of the intuitive fuzzy number. Wang et al. [19] proposed a new gray model based on data grouping and buffer operator, and the model groups on a monthly or quarterly basis are grouped, which are then buffered separately to cope with prediction error caused by seasonal fluctuations and sudden changes in trend. Li [20], by introducing the gray seasonal variation index model, combined the gray predictive method and the seasonal index to predict the seasonal demand for electricity. Shu and Dong [21] predicted tourist flow in specific regions using gray theory, respectively. Wang and $\mathrm{Li}$ [22] proposed that GDP and $\mathrm{CO}_{2}$ emissions per capita are separately regarded as the input and output to establish a nonequigap gray Verhulst model (NE gray Verhulst model) using gray systems theory. $\mathrm{Hu}$ et al. [23] made some improvements on the discrete gray model by researching the initial sensitivity problem and raw data preprocess problem of the discrete gray model. The abovementioned studies have effectively shown the advantages of the gray theory in prediction. Therefore, the gray theory is applied to predict automobile parts sales in the paper. By accumulating technology and seasonal variation index, a nonlinear periodic predictive model was built based on gray $\operatorname{GM}(1,1)$, which can dynamically predict future automobile parts sales to guide enterprises production and management according to historical sales data.

\section{Gray GM(1,1) Nonlinear Periodic Predictive Model}

2.1. Summary of Gray Theory. Many scholars have studied gray relational analysis theory based on the gray relational analysis model proposed by Professor Deng and have achieved many valuable research results [24-26]. Gray theory is used to deal with the gray element by the mathematical method, constantly deepening understanding the system and obtaining more effective information. The main contents are gray model, gray relation analysis, gray clustering, gray decision, gray control, and so on, including the theory system basis of gray set, the analysis system of gray relation space, the method system based on gray process and its generating space, and the technical system with system analysis, assessment, modelling, prediction, control, and optimization [27-29].

\subsection{Methods and Steps of Gray GM(1,1) Nonlinear Periodic} Predictive Model. The gray predictive model is an important part of gray system theory. The classical GM $(1,1)$ model is suitable for the prediction of single exponential growth, but it has some errors that are difficult to take into account the abnormal sequence data. On this basis, we construct the gray $\operatorname{GM}(1,1)$ nonlinear periodic predictive model by introducing seasonal variation index to improve the $\operatorname{GM}(1,1)$ model. The following analysis is used to build the gray $\operatorname{GM}(1,1)$ nonlinear periodic predictive model.

Definition 1. Setting $X^{(0)}=\left\{x^{(0)}(1), x^{(0)}(2), \ldots, x^{(0)}(n)\right\}$, $X^{(1)}=\left\{x^{(1)}(1), x^{(1)}(2), \ldots, x^{(1)}(n)\right\}$. So the original form of the $\operatorname{GM}(1,1)$ model is that

$$
x^{(0)}(k)+a x^{(1)}(k)=b .
$$

Definition 2. Setting $Z^{(1)}=\left\{z^{(1)}(2), z^{(1)}(3), \ldots, z^{(1)}(n)\right\}$, and $z^{(1)}(k)=(1 / 2)\left(x^{(1)}(k)+x^{(1)}(k-1)\right)$.

Then, the basic form of the $\operatorname{GM}(1,1)$ model is as follows:

$$
x^{(0)}(k)+a z^{(1)}(k)=b .
$$

Definition 3. Setting as a nonnegative sequence, $X^{(0)}$ is once accumulated generating sequence of $X^{(0)}$ (namely, 1-AGO sequence) and $Z^{(1)}$ is an immediate mean generating sequence adjacent to $X^{(0)}$, so the whitening equation of the $\mathrm{GM}(1,1)$ prediction model is

$$
\frac{d x^{(1)}}{d t}+a x^{(1)}=b
$$

where $a$ is the developmental coefficient and $b$ is the gray action. And

$$
(\mathrm{a}, \mathrm{b})^{T}=\left(B^{T} B\right)^{-1} B^{T} Y,
$$

where two matrices of $B$ and $Y$ are

$$
\begin{aligned}
& B=\left(\begin{array}{cc}
-\frac{1}{2}\left[x^{(1)}(1)+x^{(1)}(2)\right] & 1 \\
-\frac{1}{2}\left[x^{(1)}(2)+x^{(1)}(3)\right] & 1 \\
\vdots & \vdots \\
-\frac{1}{2}\left[x^{(1)}(n-1)+x^{(1)}(n)\right] & 1
\end{array}\right), \\
& Y=\left(\begin{array}{c}
x^{(0)}(2) \\
x^{(0)}(3) \\
\vdots \\
x^{(0)}(n)
\end{array}\right) .
\end{aligned}
$$




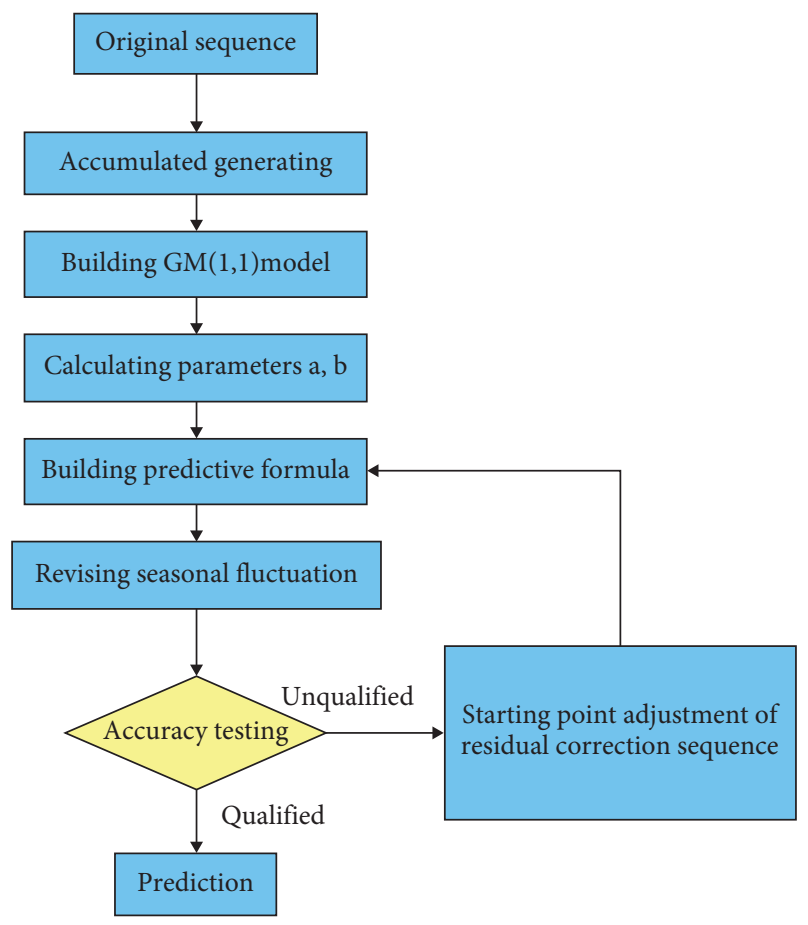

Figure 2: Steps of the gray GM(1,1) nonlinear periodic predictive model.

The $\operatorname{GM}(1,1)$ model is a differential equation that contains only one variable of time. The time series of original data acquisition is shown in Figure 1.

The steps of gray $\mathrm{GM}(1,1)$ nonlinear periodic-type predictive model are shown in Figure 2. The detailed algorithm and steps are as follows.

Step 1 (Accumulated Generation of Original Data). Accumulated data are taken as the training sample to realize the smooth sample value, which can deduce future market changes. The data time series is $X^{(0)}=\left\{x^{(0)}(1), x^{(0)}(2)\right.$, $\left.\ldots, x^{(0)}(n)\right\}$, and then the data time series is accumulated to generate new series data. That is

$$
\begin{aligned}
x^{(1)}(1) & =x^{(0)}(1), \\
x^{(1)}(2) & =x^{(0)}(1)+x^{(0)}(2), \\
x^{(1)}(3) & =x^{(0)}(1)+x^{(0)}(2)+x^{(0)}(3), \\
\ldots, &
\end{aligned}
$$

where

$$
x^{(1)}(k)=\sum_{i=1}^{k} x^{(0)}(i), \quad k=1,2, \ldots, n
$$

Step 2 (Building Predictive Equations). It is known from the gray principle that the weakening randomness of accumulated generating sequence $X^{(1)}$ has an approximate exponential growth character, which can show the growth characteristics of data, become a regular time series, and satisfy the differential equation (equation (3)). The time series response results of the predictive model can be obtained by solving the differential equation as follows:

$$
\hat{x}^{(1)}(k+1)=\left[x^{(0)}(1)-\frac{b}{a}\right] e^{-a k}+\frac{b}{a}, \quad k=1,2, \ldots, n .
$$

Step 3 (Calculation of Model Parameters). The least squares of $\operatorname{GM}(1,1)$ model parameters (equation (4)) to can be used to estimate parameters $a$ and $b$ and determine the best prediction model.

Step 4 (Restoration of data). The results of $a$ and $b$ are used to solve the predictive model (equations (3) and (9)) through cumulative reduction, restoring the original predictive series $\widehat{x}^{(0)}$ :

$$
\hat{x}^{(0)}(k+1)=\hat{x}^{(1)}(k+1)-\hat{x}^{(1)}(k), \quad k=0,1, \ldots, n .
$$

Step 5 (Introduction of the Seasonal Variation Index and Solving the Final Predictive Sales). The final predictive sales $\left(y_{k}\right)$ can be derived as follows:

$$
y_{k}=\widehat{x}^{(0)}(k) * \operatorname{SVI}_{k}, \quad k=1,2, \ldots, n .
$$

Here, the seasonal variation index is calculated by the average method of month (or quarter) as follows:

$$
\mathrm{SVI}_{k}=\frac{x_{k}}{\bar{A}}
$$

where $\mathrm{SVI}_{k}$ is the seasonal variation index, $x_{k}$ is the data value of the historical data each month (or quarter), and $\bar{A}$ is the average value of all month (or quarter).

2.3. Model Accuracy Testing. The accuracy of gray prediction model can be proved by using the residual, the correlation degree, and the posteriori difference testing [30].

2.3.1. Residual Testing. The original sequence of the variable $x^{(0)}(k)$ is $X^{(0)}=\left\{x^{(0)}(1), x^{(0)}(2), \ldots, x^{(0)}(n)\right\}$, and the corresponding fitting model sequence is $\widehat{X}^{(0)}=\left\{\widehat{x}^{(0)}(1)\right.$, $\left.\hat{x}^{(0)}(2), \ldots, \hat{x}^{(0)}(n)\right\}$. Then, the residual can be obtained as follows:

$$
\varepsilon(k)=x^{(0)}(k)-\widehat{x}^{(0)}(1), \quad k=1, \ldots, n .
$$

The relative error $\alpha_{k}=\left|\varepsilon(k) / x^{(0)}(k)\right|$, and the average relative error $\bar{\alpha}=1 / n \sum_{k=1}^{n} \alpha_{k}$; it can be obtained that $1-\alpha_{k}$ is the fitting accuracy of $k$ points and $1-\bar{\alpha}$ is the average relative accuracy.

For a given value $\alpha_{0}$, the residual model is qualified when $\bar{\alpha}<\alpha_{0}$ and $\alpha_{k}<\alpha_{0}$.

2.3.2. Correlation Degree Testing. Setting the original sequence $X^{(0)}=\left\{x^{(0)}(1), x^{(0)}(2), \ldots, x^{(0)}(n)\right\}$ and the fitting 
sequence $\widehat{X}^{(0)}=\left\{\widehat{x}^{(0)}(1), \widehat{x}^{(0)}(2), \ldots, \widehat{x}^{(0)}(n)\right\}$ and $r_{0 i}$ is an absolute correlation coefficient between $X^{(0)}$ and $\bar{X}(0)$, then

$$
\begin{array}{r}
r_{0 i}=\frac{1}{n} \sum_{k=1}^{n} r_{0 i}(k)=\frac{1}{n} \sum_{k=1}^{n} \frac{\min \left\{\Delta_{i}(k)\right\}+\rho \max \left\{\Delta_{i}(k)\right\}}{\Delta_{i}(k)+\rho \max \left\{\Delta_{i}(k)\right\}}, \\
i=1,2, \ldots, m,
\end{array}
$$

where $\Delta_{i}(k)=\left|\widehat{x}^{(0)}(k)-x^{(0)}(k)\right|$ and $\rho$ is the maximum percentage, which takes $\rho=0.5$ as usual. The relational model is qualified if $r_{0 i}>r_{0}$ for a given $r_{0 i}>0$.

2.3.3. Posteriori Difference Testing. According to the calculation, the mean square deviation of $X^{(0)}$ is

$$
S_{1}^{2}=\frac{1}{n} \sum_{k=1}^{n}\left(x^{(0)}(k)-\bar{x}\right)^{2} \text {. }
$$

And the mean square deviation of $\varepsilon(k)$ is

$$
S_{2}^{2}=\frac{1}{n} \sum_{k=1}^{n}(\varepsilon(k)-\bar{\varepsilon})^{2},
$$

where $\bar{x}$ is the mean value of $X^{(0)}, S_{1}^{2}$ is the mean square deviation, $\bar{\varepsilon}$ is the mean value of $\varepsilon(k)$, and $S_{2}^{2}$ is the mean square deviation.

(1) The mean variance ratio $c=S_{2} / S_{1}$. It is called qualified mean variance ratio model when $c<c_{0}$ for a given $c_{0}$.

(2) $p=P\left(|\varepsilon(k)-\bar{\varepsilon}|<0.6745 S_{1}\right)$ is small error probability; when $p>p_{0}$ for a given $p_{0}$, the small error probability is a qualified model.

2.3.4. Form of Model Accuracy Testing. According to the analysis, the fitting accuracy of the model prediction can be determined by the calculation of $\alpha, r_{0 i}, c$, and $p$ as shown in Table 1 [31].

\section{Empirical Analysis of Predicted Sales of the Automotive Parts}

3.1. Acquisition of the Practical Data. Data for empirical analysis are from automotive seat sales in a month of the Fcompany that is an automotive parts supplier since 2012-2018, as shown in Table 2. It can be seen that the automotive seat sales data of the F-company have a significant growth and cycle fluctuation characteristics from January 2012 to December 2018. Comparing the same month's automotive sales between 2012 and 2018, most of the same month's automotive seat sales showed an increasing trend than the one in the last year, which indicates that China's automotive part products have a good momentum of growth. The data of automotive seat sales showed that the sales were not in a linear growth. The value to turn at the seventh, eighth, and ninth month shows that the automotive sales subjected to the seasonal factors and have a certain seasonal fluctuation.
3.2. Empirical Analysis and Calculation of the Predictive Model. In the view of dual character, according to the gray GM $(1,1)$ nonlinear periodic predictive model, firstly the prediction model of automotive seat sales was established, which can reflect the sales growth trend. Secondly, the seasonal variation index was used to fit automotive seat sales of seasonal fluctuations. Finally, we form the gray $\operatorname{GM}(1,1)$ nonlinear seasonal automotive seat sales forecasting model. The detailed calculation is as follows.

Step 1 (Generation of the Accumulated Original Data and Calculation of the Seasonal Variation Index). Automotive seat sales statistics in 2012-2017 from Table 2 are taken as the sample of seat sales to predict sales in 2018, and then, the annual average of automotive seat sales is computed as the original sequence, so the original data sequence can be obtained by the average computing equation as follows:

$$
\begin{aligned}
X^{(0)}= & \left\{x^{(0)}(1), x^{(0)}(2), \ldots, x^{(0)}(6)\right\} \\
= & \{19.00333,19.23583,19.585,19.65667, \\
& 20.82917,21.35583\} .
\end{aligned}
$$

Sorting the original sequence values and eliminating the influence of extreme values in the sequence, the accumulated data are generated by using equations (7) and (8) as follows:

$$
\begin{aligned}
X^{(1)}= & \left\{x^{(1)}(1), x^{(1)}(2), \ldots, x^{(1)}(6)\right\} \\
= & \{19.00333,38.23916,57.82416, \\
& 77.48085,98.31002,119.66585\} .
\end{aligned}
$$

According to equation (12), the seasonal variation index is calculated, as shown in Table 3.

Step 2 (Establishing the Gray G(1,1) Nonlinear Periodic Predictive Model). According to equations (8) and (9), the predicted model was established. And then obtained the prediction model parameters $a$ and $b$ by equations (4), (5) and (6), where

$$
\begin{aligned}
& B=\left(\begin{array}{cc}
-\frac{1}{2}\left[x^{(1)}(1)+x^{(1)}(2)\right] & 1 \\
-\frac{1}{2}\left[x^{(1)}(2)+x^{(1)}(3)\right] & 1 \\
\vdots & \vdots \\
-\frac{1}{2}\left[x^{(1)}(5)+x^{(1)}(6)\right] & 1
\end{array}\right)=\left(\begin{array}{ccc}
-28.91208 & 1 \\
-48.03166 & 1 \\
-67.65251 & 1 \\
-87.89544 & 1 \\
-108.9879 & 1
\end{array}\right) \\
& Y=\left(\begin{array}{c}
x^{(0)}(2) \\
x^{(0)}(3) \\
\vdots \\
x^{(0)}(6)
\end{array}\right)=\left(\begin{array}{c}
19.23583 \\
19.585 \\
19.65667 \\
20.82917 \\
21.35583
\end{array}\right) .
\end{aligned}
$$


TABLE 1: Model fitting accuracy class.

\begin{tabular}{lcccc}
\hline \multirow{2}{*}{ Accuracy class } & \multicolumn{3}{c}{ Index critical value } \\
& Relative error $\alpha_{0}(\%)$ & Correlation degree $r_{0}$ & Mean variance ratio $c_{0}$ & Small error probability $p_{0}$ \\
\hline The first grade (good) & 1 & 0.90 & 0.35 & 0.95 \\
The second grade (qualified) & 5 & 0.80 & 0.50 & 0.80 \\
The third grade (possibly qualified) & 10 & 0.70 & 0.65 & 0.70 \\
The fourth grade (unqualified) & 20 & 0.60 & 0.80 & 0.60 \\
\hline
\end{tabular}

TABLE 2: Automotive seat sales of F-Company in 2012-2018 (unit: ten thousand).

\begin{tabular}{|c|c|c|c|c|c|c|c|c|c|c|c|c|}
\hline \multirow{2}{*}{ Year } & \multicolumn{12}{|c|}{ Month sales } \\
\hline & 1 & 2 & 3 & 4 & 5 & 6 & 7 & 8 & 9 & 10 & 11 & 12 \\
\hline 2012 & 15.21 & 15.03 & 18.23 & 16.54 & 20.36 & 19.63 & 13.64 & 19.05 & 24.36 & 23.15 & 20.48 & 22.36 \\
\hline 2013 & 16.14 & 15.96 & 18.63 & 16.5 & 20.15 & 19.56 & 13.42 & 19.52 & 24.65 & 23.54 & 21.35 & 21.41 \\
\hline 2014 & 16.89 & 16.52 & 19.54 & 16.34 & 20.01 & 20.82 & 14.76 & 18.06 & 25.13 & 23.68 & 21.68 & 21.59 \\
\hline 2015 & 17.05 & 16.98 & 19.15 & 16.8 & 20.08 & 20.93 & 14.07 & 18.16 & 25.52 & 23.98 & 21.51 & 21.65 \\
\hline 2016 & 17.04 & 17.06 & 17.84 & 16.35 & 20.6 & 20.41 & 15.46 & 19.42 & 26.6 & 27.37 & 24.28 & 27.52 \\
\hline 2017 & 17.11 & 17.62 & 19.56 & 16.52 & 20.89 & 21.06 & 16.58 & 20.25 & 26.72 & 27.69 & 24.39 & 27.88 \\
\hline 2018 & 18.16 & 18.10 & 20.45 & 18.12 & 22.21 & 22.14 & 16.32 & 20.89 & 27.85 & 27.41 & 24.51 & 25.91 \\
\hline
\end{tabular}

Table 3: Seasonal variation index (SVI).

\begin{tabular}{lccccccc}
\hline Month & SVI & Month & SVI & Month & SVI & Month & SVI \\
\hline 1 & 0.830981 & 4 & 0.827722 & 7 & 0.734796 & 10 & 1.24856 \\
2 & 0.828724 & 5 & 1.020258 & 8 & 0.956497 & 11 & 1.117194 \\
3 & 0.943878 & 6 & 1.022932 & 9 & 1.278393 & 12 & 1.190064 \\
\hline
\end{tabular}

By using equation (4), through the Matlab software, it can be obtained value of $a$ and $b$, respectively, that is, $a=-0.0275, b=18.2528$. If bringing $a$ and $b$ into equation (9) of the prediction model, the gray $\mathrm{G}(1,1)$ nonlinear periodic type predictive model can be determined as:

$$
\begin{aligned}
\widehat{x}^{(1)}(k+1)= & {\left[x^{(0)}(1)-\frac{b}{a}\right] e^{-a k} } \\
& +\frac{b}{a}=(19.00333+663.7382) e^{0.0275 k} \\
& -663.7382 .
\end{aligned}
$$

By Matlab, it can be known that $\hat{x}^{(1)}(k+1)$ of the predictive model as

$$
\begin{aligned}
\hat{x}^{(1)}(k+1)= & \{19.00333,38.0393,57.6059,77 . \\
& 7182,98.3912,119.6406,141.4825\},
\end{aligned}
$$

where, $k=0,1,2, \ldots, 6$.

Step 3 (Modifying Index and Make the Final Predictive Model). According to equation (10), the predicted sequence can be obtained as follows:

$$
\begin{aligned}
\widehat{x}^{(0)}(k+1)= & \widehat{x}^{(1)}(k+1)-\widehat{x}^{(1)}(k), \quad k=0,1,2,3,4,5,6 \\
= & \{19.00333,19.03597,18.9607 \\
& 20.1123,20.673,21.2493,21.8419\} .
\end{aligned}
$$

Therefore, the predicted average of automotive seat sales of the company is 21.8419 thousand in 2018. Taking into account the effect of the seasonal variation, according to equation (11) and using data of Table 3, the final sales data for the 2018 year were predicted as

$$
\begin{aligned}
y_{k}= & x(\hat{k}) * \mathrm{SVI}_{k} \\
= & \{18.15,18.10,20.62,18.08,22.28,22.34,16.05, \\
& 20.89,27.92,27.27,24.40,25.99\} .
\end{aligned}
$$

$\bar{x}(0)$

where $x(k)$ indicates the average predictive seat sales in 2018 , that is, 21.8419 .

Based on the sales data of automotive seats in the year of 2012-2017, the gray $G(1,1)$ nonlinear periodic predictive model introduced the seasonal variation index to forecast the monthly sales for the year of 2018, as shown in Table 4.

\subsection{Accuracy Test of the Predictive Model}

3.3.1. Residual Test. The residual sequence can be obtained by equation (13) as follows: $\{0.01,0,0.17,-0.04,0.07$, $0.2,-0.27,0,0.07,-0.14,-0.11,0.08\}$. And the absolute mean value of residuals is 0.097 . Then, the relative error series are $\{0.06 \%, 0 \%, 0.82 \%, 0.22 \%, 0.31 \%, 0.90 \%, 1.68 \%, 0 \%, 0.25 \%$, $0.51 \%, 0.45 \%, 0.31 \%$. And the average relative error is $0.46 \%$.

By referring to Table 1, it can be seen that the predictive model is qualified and the precision is very good, which belongs to the first grade. 
TABLE 4: Sales predictive results of automotive seats in 2018 (unit: ten thousand).

\begin{tabular}{lcc}
\hline Month and year & Actual sales $x_{(k)}^{(0)}$ & Predictive value $y_{k}$ \\
\hline Jan, 2018 & 18.16 & 18.15 \\
Feb, 2018 & 18.10 & 18.10 \\
Mar, 2018 & 20.45 & 20.62 \\
Apr, 2018 & 18.12 & 18.08 \\
May, 2018 & 22.21 & 22.28 \\
Jun, 2018 & 22.14 & 22.34 \\
Jul, 2018 & 16.32 & 16.05 \\
Aug, 2018 & 20.89 & 20.89 \\
Sept, 2018 & 27.85 & 27.92 \\
Oct, 2018 & 27.41 & 27.27 \\
Nov, 2018 & 24.51 & 24.40 \\
Dec, 2018 & 25.91 & 25.99 \\
\hline
\end{tabular}

TABLE 5: Comparison of the results of the two models (unit: ten thousand).

\begin{tabular}{lccc}
\hline Month and year & Actual value & Single GM(1,1) forecasting model predicted value & Gray GM(1,1) nonlinear periodic model predicted \\
value
\end{tabular}

3.3.2. Correlation Coefficient Test. According to equation (14), the correlation coefficient is computed, that is, $r_{0 i}=$ $0.73>0.7$. From Table 1, it can be known that this model is related to the degree of conformity and the precision grade is between the second and the third grade.

3.3.3. Posterior Residual Test. By Equation (15) and Equation (16), it is shown that

$$
\begin{aligned}
S_{1}^{2} & =13.82, \\
S_{2}^{2} & =0.0066, \\
c=\frac{S_{2}}{S_{1}} & =\frac{0.081}{3.71}=0.02<0.35 .
\end{aligned}
$$

Therefore, the predictive model of the mean variance ratio of the qualified, precision grade is the first grade and the predictive accuracy is very good.

Small error probability is $p=P\left(|\varepsilon(k)-\bar{\varepsilon}|<0.6745 S_{1}\right)=$ $1>0.95$.

By referring to Table 1, the probability of the small error of the predictive model is qualified and the predictive accuracy is very high.

In a word, the gray $\operatorname{GM}(1,1)$ nonlinear periodic predictive model is feasible to forecast the sales of automotive parts.
3.4. Comparative Analysis of Predictive Models. In order to show the advantages of the model, compared with the calculation results of the single $\operatorname{GM}(1,1)$ predictive model and the gray $\operatorname{GM}(1,1)$ nonlinear periodic type predictive model, the results are shown in Table 5.

Table 5 shows that the average relative error is $8.98 \%$, which used the gray $\operatorname{GM}(1,1)$ model to predict the automotive sales, but the proposed predictive model in the paper is only $0.46 \%$, so the error of single $\operatorname{GM}(1,1)$ forecasting model is very large and can not accurately predict the automotive seat sales. The reason is that the model of single $\operatorname{GM}(1,1)$ can only reflect the growing trend of the automotive sales, when the original data sequence $X_{(i)}^{(0)}$ does not meet the pattern of the exponential growing, and the sequence generated data of 1-AGO will not be close to the exponential growing pattern, too.

Figure 3 is the comparison result between single $\operatorname{GM}(1,1)$ forecasting model and gray $\operatorname{GM}(1,1)$ nonlinear periodic predicted model; it shows that the mean error is 1 thousand by means of the gray $\operatorname{GM}(1,1)$ nonlinear periodic predicted model. And the mean error is 22,3 thousand according to a single $\operatorname{GM}(1,1)$ forecasting model, and the error of predicted value is high. In the case of the F-company, the change rate of company's automotive seat sales is weaker than that of single $\mathrm{GM}(1,1)$ predictive model, and the predictive results are too large. The gray $\mathrm{GM}(1,1)$ nonlinear periodic predictive model modified the prediction accuracy of the single gray 


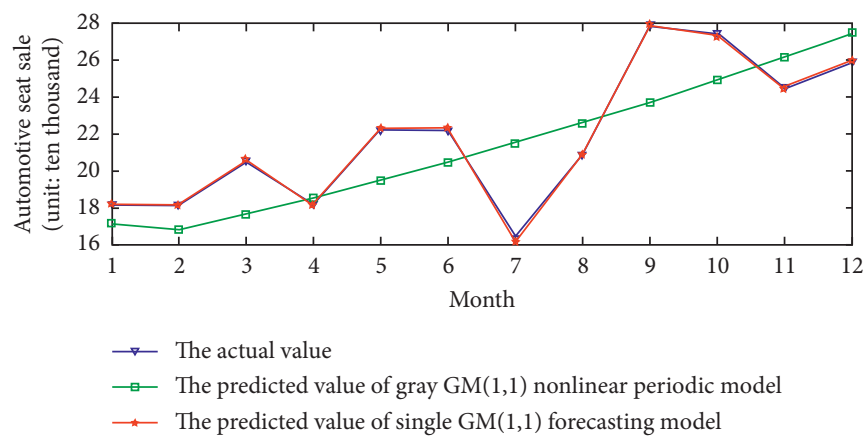

FIgURE 3: Comparison of single GM $(1,1)$ and gray $\operatorname{GM}(1,1)$ nonlinear periodic predicted model.

$\operatorname{GM}(1,1)$ model by the seasonal variation index. Most of the months' predictive values are smaller than that of the single $\mathrm{GM}(1,1)$ model. The average relative error is reduced by $8.52 \%$ compared with the single GM $(1,1)$ model.

\section{Conclusion}

The gray $\mathrm{GM}(1,1)$ nonlinear periodic predictive model is constructed by using gray theory and applied to the prediction of automotive seat. And the reliability of this model is experienced by the accuracy test, which provides a theoretical guidance for the prediction of automobile part sales. The following conclusions can be obtained:

(1) The gray $\operatorname{GM}(1,1)$ theory was studied and the fluctuation caused by the different demand of the automotive seat between off-season and busy season was considered, establishing the gray GM $(1,1)$ model. The predictive model of single $\operatorname{GM}(1,1)$ was modified by the introduction of seasonal variation index, and the accuracy of the model was verified by examples.

(2) The accuracy of the model is built on the basis of the analysis of historical data. Although it has been verified by the year of 2018, because of the limitation of the data, the accuracy of the model still needs to be improved. At the same time, the impact of the market, national policy, area, seasonal, and other factors are all influencing the sales. In the next stage of research, we would analyze the intelligent forecasting model to adapt to the changes of the market and demand and then guide the enterprise to make decisions in advance.

\section{Data Availability}

The data used to support the findings of this study are available from the corresponding author upon request.

\section{Conflicts of Interest}

The authors declare that they have no conflicts of interest.

\section{Acknowledgments}

This research was funded by the Ministry of Education of Humanities and Social Science Research Program of China
(Grant no. 15YJCZH049), Chongqing Research Program of Basic Research and Frontier Technology (Grant nos. cstc2016jcyjA0385, and cstc2017jcyjAX0343), Doctoral Scientific Research Start-up Foundation of Hubei University of Technology (Grant no.BSQD2019004), and Open Program of Academic Team of Advanced Manufacturing Technology and Equipment \& Inter Disciplinary Research Institute of Product Quality Engineering, HBUT.

\section{References}

[1] Y. X. Li and P. W. Zuo, "Short term rolling prediction method of Chinese automobile market based on seasonal adjustment model," Auto Industry Research, vol. 3, pp. 42-46, 2013.

[2] X. F. Lv, Z. X Qian, X. C. Zhang et al., "Research of China auto market composite index model based on comprehensive index method," Machine Design and Manufacturing Engineering, vol. 42, no. 1, pp. 1-12, 2013.

[3] A. T. W. Chu, R. E. Kalaba, and K. Spingarn, "A comparison of two methods for determining the weights of belonging to fuzzy sets," Journal of Optimization Theory and Applications, vol. 27, no. 4, pp. 531-538, 1979.

[4] V. Niennattrakul, P. Ruengronghirunya, and C. A. Ratanamahatana, "Exact indexing for massive time series databases under time warping distance," Data Mining and Knowledge Discovery, vol. 21, no. 3, pp. 509-541, 2010.

[5] A. Bagnall, C. A. Ratanamahatana, E. Keogh, S. Lonardi, and G. Janacek, "A bit level representation for time series data mining with shape based similarity," Data Mining and Knowledge Discovery, vol. 13, no. 1, pp. 11-40, 2006.

[6] J. M. Davis, I. A. Gravagne, B. J. Jackson, R. J. Marks II, and A. A. Ramos, "The Laplace transform on time scales revisited," Journal of Mathematical Analysis and Applications, vol. 332, no. 2, pp. 1291-1307, 2007.

[7] Q. Wu, Z. H. Yuan, and X. N. Wang, "Summary of partial least squares regression," Journal of Shenyang University, vol. 19, no. 2, pp. 33-35, 2007.

[8] S. K. Gautam and H. Om, "Multivariate linear regression model for host based intrusion detection," Computational Intelligence in Data Mining-Volume 3, vol. 33, pp. 361-371, 2015.

[9] D. J. Eck, "Bootstrapping for multivariate linear regression models," Statistics \& Probability Letters, vol. 134, pp. 141-149, 2018.

[10] W. E. Mahmod, K. Watanabe, and Ashraf A. Zahr-Eldeen, "Analysis of groundwater flow in arid areas with limited hydrogeological data using the grey model: a case study of the Nubian Sandstone, Kharga Oasis, Egypt," Hydrogeology Journal, vol. 21, no. 5, pp. 1021-1034, 2013. 
[11] T. C. Wang, M. C. Liou, and H. H. Hung, “Application of grey theory on forecasting the exchange rate between TWD and USD," in Proceedings of the Internal Conference of Business and Information, Conference Paper, pp. 14-15, Poznań, Poland, April 2005.

[12] E. Kayacan, B. Ulutas, and O. Kaynak, "Grey system theorybased models in time series prediction," Expert Systems with Applications, vol. 37, no. 2, pp. 1784-1789, 2010.

[13] Y. Zhicheng, W. Lijun, Y. Zhaokuo, and L. Haowen, "Shape optimization of welded plate heat exchangers based on grey correlation theory," Applied Thermal Engineering, vol. 123, pp. 761-769, 2017.

[14] M. S. Memon, Y. H. Lee, and S. I. Mari, "Group multi-criteria supplier selection using combined grey systems theory and uncertainty theory," Expert Systems with Applications, vol. 42, no. 21, pp. 7951-7959, 2015.

[15] X. Zeng, L. Shu, S. Yan, Y. Shi, and F. He, "A novel multivariate grey model for forecasting the sequence of ternary interval numbers," Applied Mathematical Modelling, vol. 69, pp. 273-286, 2019.

[16] Z.-X. Wang, Q. Li, and L.-L. Pei, “A seasonal GM(1,1) model for forecasting the electricity consumption of the primary economic sectors," Energy, vol. 154, pp. 522-534, 2018.

[17] Z.-X. Wang, Q. Li, and L.-L. Pei, "Grey forecasting method of quarterly hydropower production in China based on a data grouping approach," Applied Mathematical Modelling, vol. 51, pp. 302-316, 2017.

[18] P. Li, S. F. Liu, and J. J. Zhu, "GM(1,1) prediction model based on intuitionistic fuzzy number," Control and Decision, vol. 28, no. 10, pp. 1583-1586, 2013.

[19] Z.-X. Wang, L.-Y. He, and H.-H. Zheng, "Forecasting the residential solar energy consumption of the United States," Energy, vol. 178, pp. 610-623, 2019.

[20] S. Li, "Research on electricity demand forecasting method based on improved grey GM mode," Power Demand Side Management, vol. 8, no. 5, pp. 17-19, 2006.

[21] L. J. Shu and W. Dong, "Forecast on Jin Cheng Shan tourism passenger based on the $\operatorname{GM}(1,1)$ model," GanSuNongYe, vol. 24, no. 9, pp. 19-20, 2011.

[22] Z.-X. Wang and Q. Li, "Modelling the nonlinear relationship between $\mathrm{CO}_{2}$ emissions and economic growth using a PSO algorithm-based grey Verhulst model," Journal of Cleaner Production, vol. 207, pp. 214-224, 2019.

[23] R. Hu, Q. J. Zhuang, L. Zhu, and Y. Fu, "Application of improved discrete grey model in medium-long term power load forecasting," Journal of Electric Power Science and Technology, vol. 24, pp. 50-53, 2009.

[24] S. F. Liu, N. M. Xie, and F. Jeffery, "On new models of grey incidence analysis based on visual angle of similarity and nearness," Systems Engineering-Theory \& Practice, vol. 30, no. 5, pp. 881-887, 2010.

[25] Y. G. Dang, S. F. Liu, B. Liu et al., "Study on the weakening buffer operators and their applications," in Proceedings of the 7th International Conference on Industrial Management, Okayama, Japan, October 2004.

[26] J. L. Deng, "Introduction to grey system theory," The Journal of Grey System, vol. 1, no. 1, pp. 1-24, 1989.

[27] S. F. Liu and J. Forrest, "The role and position of grey system theory in science development," The Journal of Grey System, vol. 9, no. 4, pp. 351-356, 1997.

[28] S. F. Liu and J. Forrest, "Advances in grey systems theory and its applications," in Proceedings of the 2007 IEEE International Conference on Grey Systems and Intelligent Services, Nanjing, China, May 2007.
[29] S. F. Liu, H. Cai, Y. Cao, and Y. J. Yang, "Advance in grey incidence analysis modeling," in Proceedings of the 2011 IEEE International Conference on Systems, Man, and Cybernetics, vol. 33, no. 8, pp. 2041-2046, Anchorage, AK, USA, November 2013.

[30] L. L. Ma, G. Li, and D. Q. Tao, "A failure prediction method based on gray GM(1,1) model," Computer Applications and Software, vol. 30, no. 4, pp. 198-200, 2013.

[31] C. Q. Yuan, S. F. Liu, and Z. G. Fang, "Comparison of China's primary energy consumption forecasting by using ARIMA (the autoregressive integrated moving average) model and GM(1,1) model," Energy, vol. 100, pp. 384-390, 2016. 


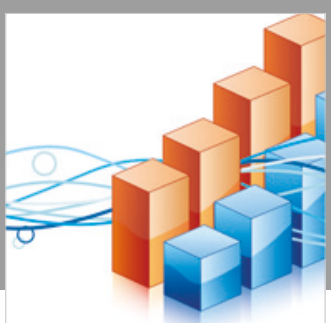

Advances in

Operations Research

\section{-n-m}
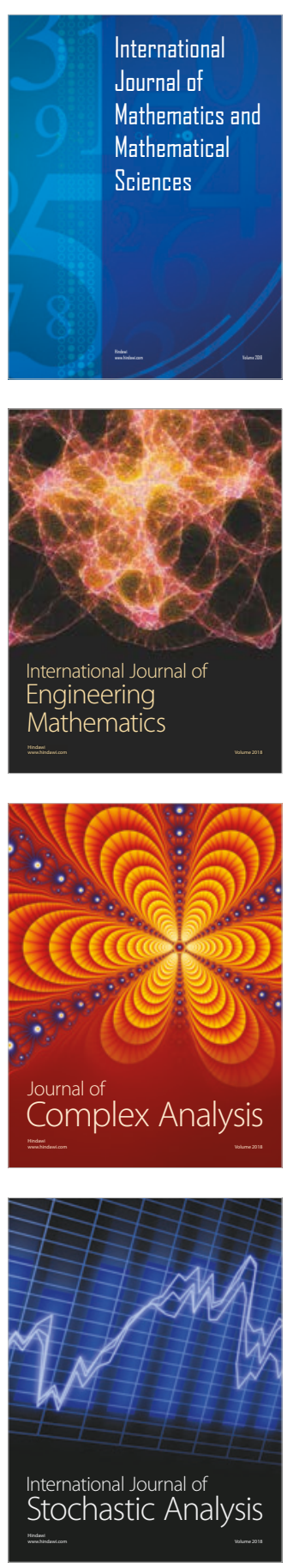
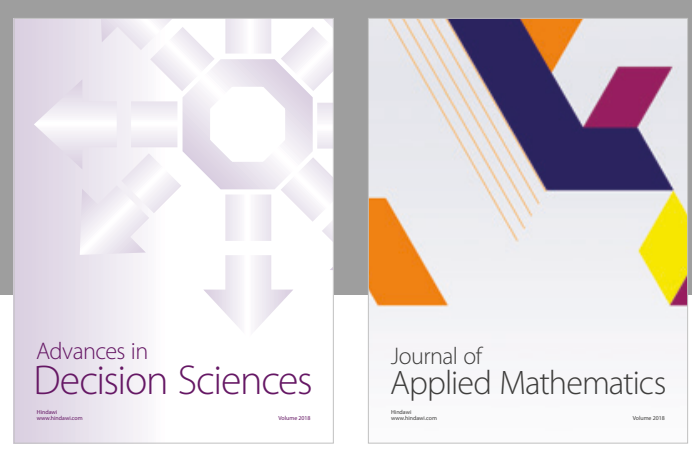

Journal of

Applied Mathematics
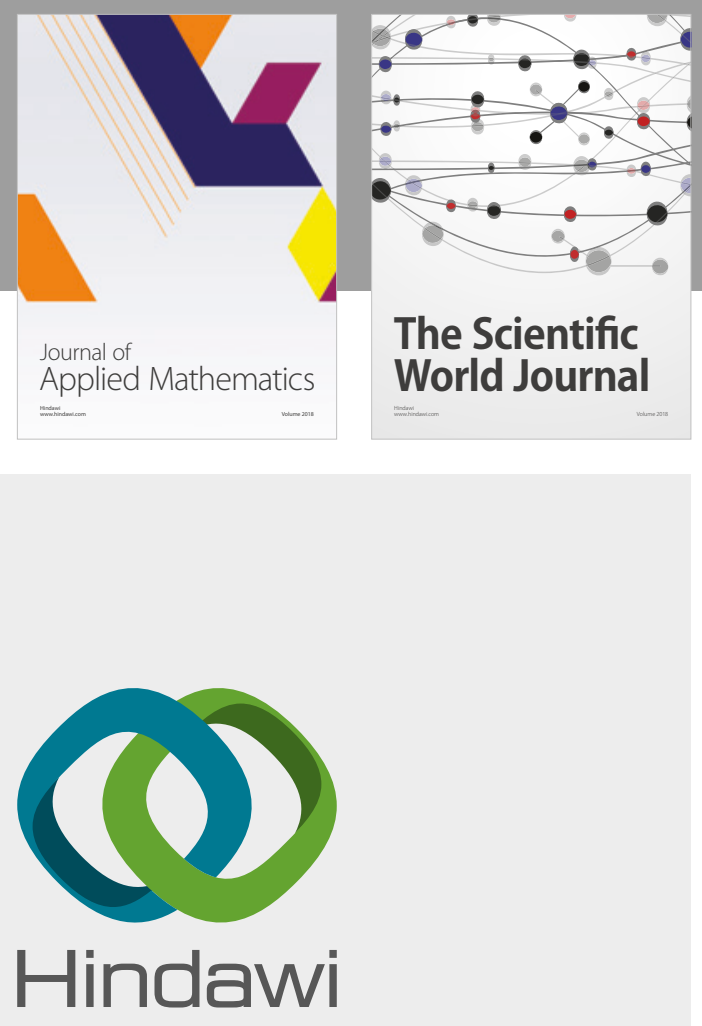

Submit your manuscripts at

www.hindawi.com

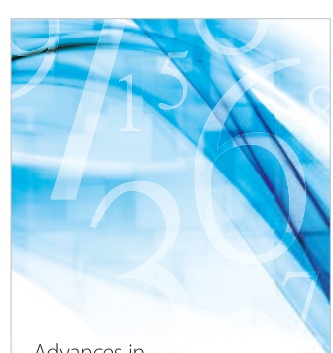

Advances in
Numerical Analysis
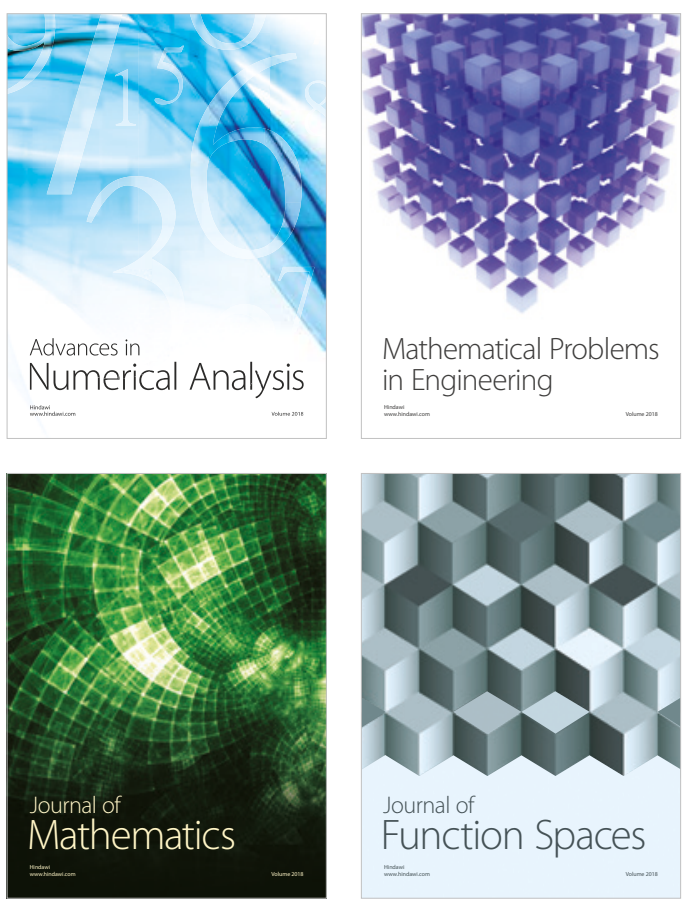

Mathematical Problems in Engineering

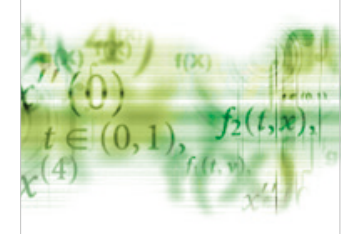

International Journal of

Differential Equations

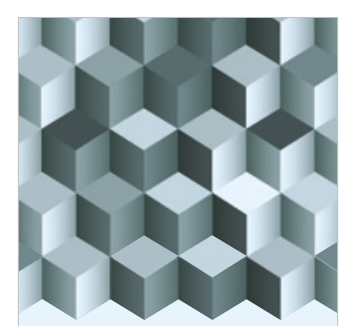

Journal of

Function Spaces

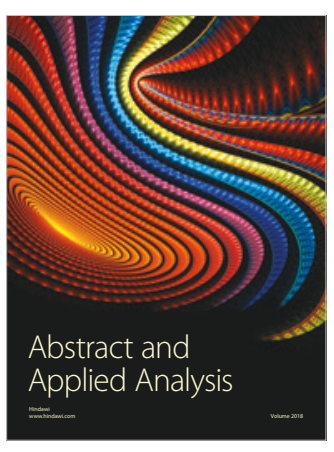

The Scientific

World Journal

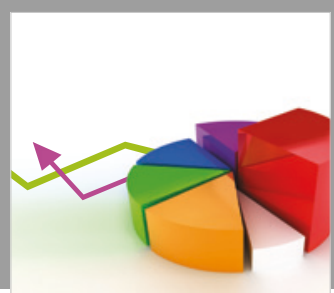

Journal of

Probability and Statistics
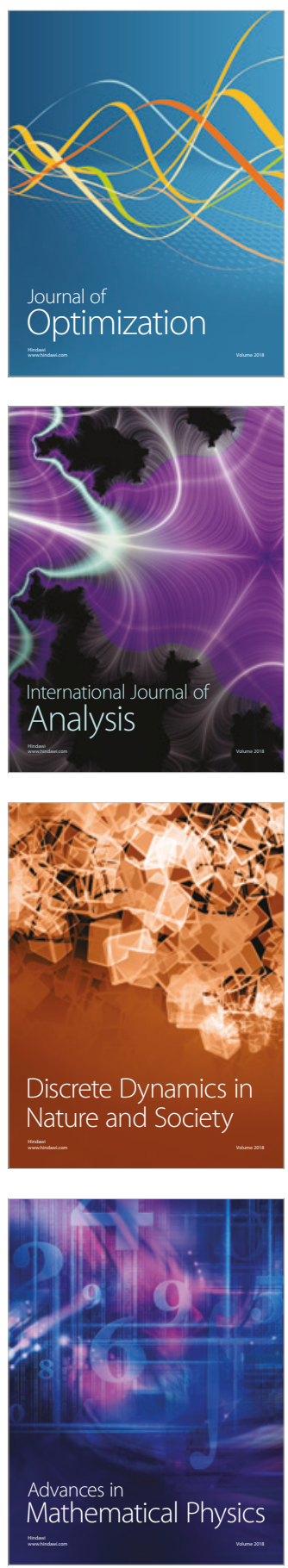